\title{
BMJ Open Dual antiplatelet therapy following percutaneous coronary intervention: protocol for a systematic review
}

\author{
Jesse Elliott, ${ }^{\oplus 1,2}$ Shannon E Kelly, ${ }^{1,2}$ Zemin Bai, ${ }^{1}$ Becky Skidmore, ${ }^{3}$ \\ Michel Boucher, ${ }^{4}$ Derek Y F So, ${ }^{5}$ George A Wells ${ }^{1,2}$
}

To cite: Elliott J, Kelly SE, Bai Z, et al. Dual antiplatelet therapy following percutaneous coronary intervention: protocol for a systematic review. BMJ Open 2019;9:e022271. doi:10.1136/ bmjopen-2018-022271

- Prepublication history and additional material for this paper are available online. To view these files, please visit the journal online (http://dx.doi. org/10.1136/bmjopen-2018022271).

Received 12 February 2018 Revised 27 December 2018 Accepted 8 January 2019

\section{Check for updates}

(C) Author(s) (or their employer(s)) 2019. Re-use permitted under CC BY-NC. No commercial re-use. See rights and permissions. Published by BMJ.

${ }^{1}$ Cardiovascular Research Methods Centre, University of Ottawa Heart Institute, Ottawa, Ontario, Canada

${ }^{2}$ School of Epidemiology and

Public Health, University of

Ottawa, Ottawa, Ontario, Canada

${ }^{3}$ Independent Information

Specialist, Ottawa, Ontario,

Canada

${ }^{4}$ Program and Policy

Development, Canadian Agency

for Drugs and Technologies in

Health (CADTH), Ottawa, Ontario,

Canada

${ }^{5}$ University of Ottawa Heart

Institute, Ottawa, Ontario,

Canada

Correspondence to

Professor George A Wells;

gawells@ottawaheart.ca

\section{ABSTRACT}

Introduction Dual antiplatelet therapy (DAPT) is routinely given to patients after percutaneous coronary intervention (PCl) with stenting; however, there is ongoing debate about the optimal duration, especially in specific patient groups. In the proposed systematic review, we intend to assess the optimal duration of DAPT following PCI with stenting, with a focus on clinically relevant patient subgroups.

Methods and analysis We will perform a comprehensive search of the published literature for randomised controlled trials (RCTs) assessing the benefits and harms of extended DAPT (>12 months) compared with shortterm DAPT (6-12 months) following PCl with stenting (bare metal or drug eluting). ClinicalTrials.gov and ICTRP will also be searched to identify ongoing and completed clinical trials. Two independent reviewers will select studies for inclusion, and the risk of bias will be assessed by use of Cochrane's Risk of Bias tool. The primary outcome of interest is death (all-cause, cardiovascular, non-cardiovascular). Secondary outcomes are bleeding (major, minor, gastrointestinal), urgent target vessel revascularisation, major adverse cardiovascular events, myocardial infarction, stroke and stent thrombosis. Subgroup data will be sought for patients with prior myocardial infarction, acute coronary syndrome at presentation and diabetes, and based on smoking status and age group. Data will be analysed by random-effects meta-analysis, and separate analyses will be performed for patient subgroups. Bayesian network meta-analysis will be performed to investigate the effect of individual P2Y12 inhibitors at different DAPT durations longer than 6 months.

Ethics and dissemination This review will provide a comprehensive overview of the available evidence of the benefits and harms associated with extending DAPT beyond 12 months following $\mathrm{PCl}$ with stenting and the effects on clinically important subgroups. The results of this review will inform clinical and policy decisions regarding the optimal treatment duration of DAPT following $\mathrm{PCl}$ with stenting.

Systematic review registration PROSPERO no. CRD42018082587

\section{INTRODUCTION}

Current guidelines recommend that patients be given dual antiplatelet therapy (DAPT; combination of a P2Y12 inhibitor with

\section{Strengths and limitations of this study}

This review builds on our previous umbrella review of systematic reviews by focusing on clinically relevant patient subgroups.

- This protocol was designed following methods of the Cochrane Handbook for Systematic Reviews for Interventions and is reported following the Preferred Reporting Items for Systematic review and MetaAnalyses for Protocols guidelines.

- Limitations may be due to heterogeneity in outcome and subgroup definitions across included trials. This will be overcome by pooling only outcome data with consistent definitions.'

acetylsalicylic acid) ranging from 6 to 12 months following percutaneous coronary intervention (PCI) with stenting, with the aim of preventing stent thrombosis and major adverse cardiovascular events (MACCE). ${ }^{1-4}$ However, debate is ongoing about the optimal duration of DAPT; importantly, patient characteristics may be an important factor in duration decision. ${ }^{5}$ In some settings, DAPT for even less than 6 months may be appropriate (eg, patients with high risk of bleeding), while other patients may derive greater benefit from extended DAPT (eg, high risk of stent thrombosis and low risk of bleeding). ${ }^{4}$ Previous reviews have reported an increased risk of death among patients who received DAPT for more than 12 months following stenting, ${ }^{67}$ but whether this risk is common across all patient subgroups is unclear.

Previous systematic reviews have attempted to determine the optimal duration of $\mathrm{DAPT}^{6-16}$; however, few have examined the impact of specific patient characteristics or type of P2Y12 inhibitor on the effect estimate. Navarese and colleagues ${ }^{7}$ reported a reduced risk of definite or probable stent thrombosis in patients without, but not with, acute coronary syndrome (ACS) who received extended DAPT compared with 12 months of DAPT; 
however, no significant differences were reported in the risk of cardiovascular death or myocardial infarction. A recent study by Shah and colleagues ${ }^{17}$ that focused on network meta-analysis found that, among patients randomised to ticagrelor, prasugrel or clopidogrel, the risk of major adverse cardiac events and myocardial infarction (MI) were lower with both ticagrelor and prasugrel compared with clopidogrel. Shah and colleagues ${ }^{17}$ reported a reduced risk of all-cause and cardiovascular death among patients randomised to ticagrelor compared with clopidogrel. However, whether these results are consistent at all durations of DAPT is unknown. Our review will build on earlier reviews by providing an up-to-date evidence synthesis for a wide range of patient subgroups and by including additional clinically relevant outcomes.

To make appropriate decisions, clinicians require a transparent and comprehensive review of the evidence to evaluate the potential benefits and harms associated with extending DAPT beyond 12 months after stenting for potentially personalising therapy for individual patients. In this study, we will perform a systematic review to assess the benefits and harms associated with extended DAPT following PCI with stenting. In addition to pooling the data for all patients, we will investigate the effect of extended DAPT in clinically relevant patient subgroups, including age, history of MI, ACS at presentation, diabetes and smoking status, and the impact of individual P2Y12 inhibitors.

\section{METHODS AND ANALYSES}

This review has been registered PROSPERO (CRD42018082587) and follows the Preferred Reporting Items for Systematic Reviews and Meta-Analyses Protocols (PRISMA-P) guidelines. ${ }^{18}$ The completed PRISMA-P checklist is available (online supplementary file 1). The review will follow the methods of the Cochrane Handbook for Systematic Reviews for Interventions ${ }^{19}$ and the PRISMA checklist for systematic reviews. ${ }^{20}$

\section{Search strategy}

An experienced medical information specialist developed and tested the strategy using an iterative process in consultation with the review team. Using the Ovid platform, we searched Embase and Ovid MEDLINE, including Epub Ahead of Print and In-Process \& Other Non-Indexed Citations. We also searched the Cochrane Library as well as PubMed for the most recent and unindexed citations only. The search strategies utilised a combination of controlled vocabulary (eg, 'Stents', 'Percutaneous Coronary Intervention', 'Purinergic P2Y Receptor Antagonists') and keywords (eg, 'DES', 'PCI', 'dual antiplatelet therapy'). Vocabulary and syntax were adjusted across databases. An amended version of the Cochrane highly sensitive search strategy was applied to identify randomised controlled trials. ClinicalTrials.gov and the ICTRP search portal will also be searched to identify ongoing and completed clinical trials. No language or date restrictions were applied. The search strategy is shown in online supplementary file 2.

\section{Inclusion criteria}

The inclusion criteria for this review will follow the PICOS (population, intervention, comparison, outcome, study design) criteria:

\section{Population}

Adult patients who have undergone PCI with any type of stent and who are receiving DAPT. Patients receiving DAPT in the absence of stenting are beyond the scope of this review, and studies involving less than $85 \%$ of patients who underwent stent implantation will be excluded.

\section{Intervention}

DAPT following PCI with stenting for an extended duration (>12 months). DAPT may involve any type of P2Y12 inhibitor (clopidogrel, prasugrel, ticagrelor) in combination with acetylsalicylic acid.

\section{Comparison}

DAPT for 6 to 12 months. The comparison of DAPT for less than 6 months to more than 12 months is beyond the scope of this review.

\section{Outcomes}

The primary outcome of interest is death (all-cause, cardiovascular, non-cardiovascular). The secondary outcomes are urgent target vessel revascularisation, MACCE, MI, stroke, stent thrombosis, and major, minor and gastrointestinal bleeding, as defined by the individual study protocols and/or publications. A range of bleeding classifications and MACCE definitions are expected, and data will be analysed separately by classification type or definition. Studies will not be included or excluded on the basis of reported outcomes.

\section{Study design}

Randomised controlled trials.

\section{Study selection}

Two independent reviewers will apply the eligibility criteria based on the PICOS to each title and abstract identified in the literature search. All records deemed potentially relevant by at least one will be obtained in full-text format. The eligibility criteria will be applied to the full-text records by both reviewers independently, and a final decision about eligibility will be made. Conflicts will be resolved by discussion. The reviewers will not be blinded to study authors or centre of publication prior to study selection. Study screening and assessment of eligibility will be facilitated and standardised through the use of DistillerSR (Evidence Partners), an online systematic review software.

\section{Quality assessment}

Two independent reviewers will apply the Cochrane Collaboration's Risk of Bias tool to each included RCT, and any disagreements will be resolved by discussion. 


\section{Data extraction}

Data will be extracted by one reviewer by use of piloted and standardised data abstraction forms, and the extracted data will be checked for accuracy by a second reviewer. Any disagreements will be resolved by consensus. The original, primary publication for each included RCT will be used for data extraction, with supplementary data obtained from companion reports where necessary to address the research questions and/or contacting authors for additional subgroup information. Multiple publications for a unique RCT (eg, supplemental online appendices, companion publications of specific outcomes or populations from the original study) will be handled by extracting the most recently adjudicated data for each outcome specified a priori in this protocol.

Data to be extracted include study characteristics (eg, author, year, study design, country of study) and participant characteristics (eg, age, sex, smoking status, diabetes, prior MI, presence of ACS at presentation, history of heart failure). Intervention and comparator details (eg, DAPT duration, type of P2Y12 inhibitor) and outcome data at the longest duration of follow-up will also be extracted.

\section{Subgroups}

Effect estimates will be extracted separately for clinically important subgroups (ie, age, history of MI, ACS at presentation, diabetes and smoking status). If available, data will also be extracted separately for type of P2Y12 inhibitor (clopidogrel, prasugrel, ticagrelor).

\section{Data synthesis}

A descriptive summary of study selection, quality assessment and study and patient characteristics will be presented. We will assess clinical heterogeneity by examining the patient characteristics of the included studies, and methodological heterogeneity by assessing the study design characteristics. Statistical heterogeneity will be assessed by use of the $I^{2}$ statistic, with $I^{2}$ values above $75 \%$ considered to represent substantial heterogeneity; pooled data will not be reported above this threshold. If data are insufficient or if high heterogeneity is detected, descriptive summaries will be presented. If data are sufficient in quantity and sufficiently similar, random-effects pair-wise meta-analysis will be undertaken by use of RevMan (V.5.3; Cochrane Collaboration). The relative risk and $95 \%$ CI for each outcome will be determined (ie, >12 months of DAPT versus 6-12 months of DAPT). The number of participants randomised to each group will be used as the denominator for all analyses, while the number of participants who experienced each outcome will form the numerator. Study-level outcome data will be analysed separately for each subgroup, according to the subgroup definition reported by the included trials (eg, with or without diabetes). Age will be dichotomised as younger or older than 75 years. If sufficient data are available, Bayesian network meta-analysis will be undertaken to analyse the effect of individual P2Y12 inhibitors at different durations of DAPT. A continuity correction will be applied to adjust for zero events. Assessment of model fit and choice of model (fixed vs random effects) will be based on the deviance information criterion and comparison of residual deviance to the number of unconstrained data points. ${ }^{21}$ Point estimates and $95 \%$ credible intervals will be calculated by use of the Markov chain Monte Carlo method. Vague priors (eg, $\mathrm{N}\left[0,100^{2}\right]$ ) will be assigned for basic parameters, ${ }^{21}$ and trace plots and Brooks-GelmanRubin statistics will be assessed for model convergence. ${ }^{22}$ Three chains will be fit with at least 20000 iterations and a burn-in of at least 20000 iterations. Where possible, inconsistency will be assessed by comparing the deviance, between-study variance, and deviance information criterion statistics of the consistency and inconsistency models. ${ }^{23}$ Network evidence diagrams will be constructed by use of NodeXL, and network meta-analyses will be performed by use of WinBUGS (v.1.4.3; MRC Biostatistics Unit).

\section{Patient and public involvement}

Patients and the public were not involved in the development of this protocol.

\section{ETHICS AND DISSEMINATION}

In this systematic review, we will undertake a comprehensive review of the evidence supporting the optimal duration of DAPT, with an emphasis on the benefits and harms of extended DAPT in clinically relevant patient subgroups. These results will be of interest to clinicians, policy makers, and patients. Results will be disseminated through a peer-reviewed publication and conference presentations. Ethics approval is not required for this study.

Contributors JE, SEK, ZB, MB, DS and GAW designed the study. BS designed and executed the search strategy. JE drafted the protocol, which was revised by all authors. All authors have approved the version of the manuscript submitted for publication.

Funding This research was funded by CADTH (Ottawa, Ontario), an independent organisation established and funded by the federal, provincial and territorial governments in Canada. CADTH is responsible for providing Canada's healthcare decision-makers with objective evidence about the optimal use of drugs and medical devices, and provided input into the conception and design of this study, in order to ensure relevance to their jurisdictional policy questions.

Competing interests None declared.

Patient consent for publication Not required.

Provenance and peer review Not commissioned; externally peer reviewed.

Open access This is an open access article distributed in accordance with the Creative Commons Attribution Non Commercial (CC BY-NC 4.0) license, which permits others to distribute, remix, adapt, build upon this work non-commercially, and license their derivative works on different terms, provided the original work is properly cited, appropriate credit is given, any changes made indicated, and the use is non-commercial. See: http://creativecommons.org/licenses/by-nc/4.0/.

\section{REFERENCES}

1. Tanguay JF, Bell AD, Ackman ML, et al. Focused 2012 update of the Canadian Cardiovascular Society guidelines for the use of antiplatelet therapy. Can J Cardiol 2013;29:1334-45. 
2. The Task Force on Myocardial Revascularization of the European Society of Cardiology (ESC) and the European Association for Cardio-Thoracic Surgery (EACTS). 2014 ESC/EACTS Guidelines on myocardial revascularization. Eur Heart J 2014;35:2541-619.

3. Valgimigli M, Bueno H, Byrne RA, et al. 2017 ESC focused update on dual antiplatelet therapy in coronary artery disease developed in collaboration with EACTS: the task force for dual antiplatelet therapy in coronary artery disease of the European Society of Cardiology (ESC) and of the European Association for Cardio-Thoracic Surgery (EACTS). Eur Heart J 2018;39:213-60.

4. Levine GN, Bates ER, Mauri L, et al. Guideline focused update on duration of dual antiplatelet therapy in patients with coronary artery disease a report of the American College of Cardiology / American heart association task force on clinical practice guidelines. Circulation 2016:133.

5. Wells GA, Elliott J, Kelly S, et al. Dual Antiplatelet Therapy following Percutaneous Coronary Intervention: a Review of the Clinical Impact of Treatment Duration. University of Ottawa: Ottawa, ON, 2017. https://cadth.ca/sites/default/files/pdf/HT0001_DAPT_Post_PCl_. pdf. [Accessed 11 Feb 2019].

6. Fei Y, Tsoi MF, Cheung TT, et al. Optimal duration of dual antiplatelet therapy after drug-eluting stent implantation: meta-analysis of randomized controlled trials. Int J Cardiol 2016;220:895-900.

7. Navarese EP, Andreotti F, Schulze V, et al. Optimal duration of dual antiplatelet therapy after percutaneous coronary intervention with drug eluting stents: meta-analysis of randomised controlled trials. BMJ 2015;350:h1618.

8. Zhang X-L, Zhu Q-Q, Zhu L, et al. Optimize the duration of DAPT following DES implantation: an updated system review and metaanalysis of 10 randomized trials. Clin Trials Regul Sci Cardiol 2015;6:1-11.

9. Xie C, Ding XL, Miao LY. Different durations of dual anti-platelet therapy after percutaneous coronary intervention with drug-eluting stents in patients with coronary disease: a systematic review. Chinese Pharm. J 2016;51:762-8.

10. Verdoia M, Schaffer A, Barbieri L, et al. Optimal duration of dual antiplatelet therapy after DES implantation: a meta-analysis of 11 randomized trials. Angiology 2016;67:224-38.

11. Tsoi MF, Cheung CL, Cheung TT, et al. Duration of dual antiplatelet therapy after drug-eluting stent implantation: meta-analysis of large randomised controlled trials. Sci Rep 2015;5:13204.
12. Palmerini T, Benedetto $U$, Bacchi-Reggiani L, et al. Mortality in patients treated with extended duration dual antiplatelet therapy after drug-eluting stent implantation: a pairwise and Bayesian network meta-analysis of randomised trials. Lancet 2015;385:2371-82.

13. Palla M, Briasoulis A, Siddiqui $F$, et al. Long ( $>12$ months) and short ( $<6$ months) versus standard duration of dual antiplatelet therapy after coronary stenting: a systematic review and meta-analysis. $A m \mathrm{~J}$ Ther 2017;24:e468-76.

14. Cassese S, Byrne RA, Ndrepepa G, et al. Prolonged dual antiplatelet therapy after drug-eluting stenting: meta-analysis of randomized trials. Clin Res Cardiol 2015;104:887-901.

15. Bittl J, Baber U, Bradley S, et al. Duration of dual antiplatelet therapy: a systematic review for the 2016 ACC/AHA guideline focused update on duration of dual antiplatelet therapy in patients with coronary artery disease: a report of the American College of Cardiology/ American Heart Association. Circulation 2016;68:1116.

16. D'Ascenzo F, Moretti C, Bianco M, et al. Meta-analysis of the duration of dual antiplatelet therapy in patients treated with secondgeneration drug-eluting stents. Am J Cardiol 2016;117:1714-23.

17. Shah R, Rashid A, Hwang I, et al. Meta-analysis of the relative efficacy and safety of oral P2Y12 inhibitors in patients with acute coronary syndrome. Am J Cardiol 2017;119:1723-8.

18. Moher $D$, Shamseer $L$, Clarke $M$, et al. Preferred reporting items for systematic review and meta-analysis protocols (PRISMA-P) 2015 statement. Syst Rev 2015;4:1.

19. Higgins JP, Green S. Cochrane Handbook for Systematic Reviews of Interventions. UK: Wiley-Blackwell, 2008.

20. Moher D, Liberati A, Tetzlaff J, et al. Preferred reporting items for systematic reviews and meta-analyses: the PRISMA statement. PLOS Med 2009;6:e1000097.

21. Dias S, Sutton A, Welton N, et al. NICE DSU technical support document 2: generalised linear modelling framework for pairwise and network meta-analysis of randomised controlled trials. www.nicedsu. org.uk/TSD6 Software.final.08.05.12.pdf

22. Spiegelhalter $\mathrm{D}$, Thomas $\mathrm{A}$, Best $\mathrm{N}$, et al. WinBUGS user manual. 2003 www.mrc-bsu.cam.ac.uk/wp-content/uploads/manual14.pdf

23. Dias $\mathrm{S}$, Welton NJ, Sutton AJ, et al. Evidence synthesis for decision making 4: inconsistency in networks of evidence based on randomized controlled trials. Med Decis Making 2013;33:641-56. 